\title{
We E05
}

Broadband Data - Integration of the Knowledge

D. Sineva* (Repsol Sinopec Brasil)

\section{SUMMARY}

Broad Band technology used for seismic data acquisition opened many possibilities for detail data interpretation and reservoir characterization in a complex areas where resolution is necessary. During the last few years our industry saw many examples of fantastic dataset with significant data quality improve in terms of signal to noise ratio, vertical and lateral resolution and etc..

Is this enough to assume that Broad Band data by definition will provide the superior data quality? Is there an additional work that should be done to guarantee that results will meet the expectations? And how should we set those expectation? These are some of many questions we need to find answers before we commit to broad band or any kind of new technologies. 